\title{
Defining the role of curative local therapy in oligometastatic cancer: a new era
}

Oligometastatic cancer is defined as a clinical state of metastatic disease that is limited to "oligo" or few metastatic lesions as identified by imaging. Conceptualized in the 1990s by Drs. Hellman and Weichselbaum, it has now become an accepted condition in the natural history of most solid malignancies. Historically, the primary approach of metastatic cancer treatment has been prolonging disease control with systemic therapy and palliating symptoms with medical management and radiotherapy. As the oligometastatic state moves beyond a hypothesis, questions on the optimal treatment and ultimate goals of care (including potential for cure) for these patients will arise. Unlike in widespread metastatic disease, where systemic therapy plays a pivotal role in controlling the systemic disease burden, for oligometastatic disease, the role of metastasisdirected local therapy with radiation therapy or surgery, in conjunction with systemic therapy, is crucial. Treatment in this situation has the potential to improve provide durable local control, delay the time to next-line systemic therapy, and even prolong survival, possibly with cure, by eradicating visible disease burden. This focused series, with ten timely and impactful articles, addresses the concept of oligometastases, the current status of published literature on the treatment of oligometastases, and the role of ablative radiation therapy for oligometastatic disease for various cancers.

The first article discusses the concept of oligometastases, and the history from which the hypothesis of the oligometastatic disease arose (1). A detailed discussion on the technical aspect of delivering such an ablative dose of radiotherapy for oligometastatic disease (2) sets the stage for the subsequent articles that delve into the treatment of oligometastases from specific cancer types or sites. The article by Mihai et al. provides a detailed description of the technology using linear accelerator (LINAC)-based stereotactic body radiation therapy (SBRT) [or stereotactic ablative radiotherapy (SABR)], and the necessary steps to develop a program where such service can be provided.

Lung cancer is the leading cause of cancer related mortality globally. While immunotherapy (IO) has made significant improvements in the outcomes of advanced non-small cell lung cancer (NSCLC), response rates to IO remain only modest, and the large majority of patients with advanced NSCLC die from their disease. Dohopolski et al. (3) review the treatment of oligometastases from NSCLC and how recent evidence from prospective studies have shown a survival benefit from using SBRT in this setting. With significant advancements in systemic therapy for breast cancer, a rationale combination of systemic therapy with locally ablative therapy for oligometastases carries more importance. Kent et al. (4) provide a detailed review of the role of SBRT in oligometastatic breast cancer. While there has been significant advancement made in curing localized prostate cancer using surgery and radiation therapy, many patients develop metastatic disease. Androgen deprivation remains the mainstay of initial therapy for metastatic prostate cancer, but eventually the disease becomes hormone refractory. The article by Sritharan et al. (5) reviews the current evidence not only for SBRT in oligometastatic prostate cancer but also usefulness of prostate-directed therapy in the presence of untreated prostate cancer with synchronous oligometastatic disease.

One of the early success stories of treating oligometastatic cancer combining both effective systemic therapy and local treatment was in colorectal cancers with liver metastasis found synchronously at the time of initial diagnosis. Chandy et al. (6) provide a historical overview of hepatic resection in the setting of de novo oligometastatic colorectal cancer. In addition, they provide a detailed description of management of these complex scenarios in the multidisciplinary setting, which is a key to the success of such an approach.

While in adults the treatment of oligometastatic cancer has gained a momentum, the concept and management of pediatric patients with oligometastatic disease are evolving. The next article by Smile et al. (7) provides an overview of current and future research in pediatric cancers with oligometastatic disease. Given that the pediatric population is particularly vulnerable to long-term radiation-induced toxicities, this article provides information on available evidence of such toxicities when an aggressive ablative radiation treatment approach is utilized.

The brain is one of the most common sites of cancer spread. Mounting data over the past two decades have shown that single-fraction stereotactic radiotherapy (SRS) can successfully control limited brain metastasis. The omission of whole brain radiation therapy does not impact survival and results in improved neuro-cognitive outcomes. The article by Chaung et al. (8) reviews the management of limited brain metastasis utilizing a stereotactic approach. The article also describes future 
directions in studying SRS for patients with $>10$ brain metastasis as well as the potential effects of newer systemic therapy strategies in combination with SRS.

Looking to the future of oligometastatic disease management, the article by Turchan et al. (9) explores the role of IO in combination with SBRT. As clinical trials demonstrate benefits of IO for specific cancers, it will become important to explore and understand the role of radiation in combination with IO. Despite the successes of IO, the response rate is limited and many patients with initial response eventually progress. With ablative radiotherapy, there is a potential to augment the effect of IO, including with the potential for an abscopal effect (i.e., unanticipated response in lesions not targeted with radiation).

Finally, the article by Li et al. (10) summarizes ongoing clinical trials using SBRT in the oligometastatic setting.

In summary, stereotactic radiotherapy is an established technique of delivering ablative doses effectively and safely. Combining such ablative radiation with conventional systemic drugs and newer agents like IO is an active area of research for multiple cancers. The articles in this focused series provide the current evidence on the concept of oligometastatic disease and the use of SBRT for oligometastases. Additionally, future directions, including ongoing clinical trials, are highlighted throughout this special series. To better understand the oligometastatic state and the treatments that can optimally manage patients with oligometastases, further research is needed, with a focus on identifying those factors that best predict response to treatment and determining the optimal candidates for metastasis-directed local therapy.

\section{Acknowledgments}

Funding: None.

\section{Footnote}

Provenance and Peer Review: This article was commissioned by the editorial office, Annals of Palliative Medicine for the series "Oligometastasis-Fallacy or Real Deal?". The article did not undergo external peer review.

Conflict of Interest: All authors have completed the ICMJE uniform disclosure form (available at http://dx.doi.org/10.21037/ apm-2021-02). The series "Oligometastasis- Fallacy or Real Deal?" was commissioned by the editorial office without any funding or sponsorship. Dr. CBS serves as an unpaid Editor-in-Chief of Annals of Palliative Medicine. Dr. SSL serves as an unpaid Associate Editor-in-Chief of Annals of Palliative Medicine from Dec 2019 to Nov 2021. All authors served as the unpaid Guest Editors of the series. Dr. MTM reports royalties from Wolters Kluwer, consulting fees from Galera Therapeutics and speaker fees from Astra Zeneca. Dr. TB reports royalties from Demos Medical, consulting fees from Galera Therapeutics and speaker fees from Astra Zeneca. Dr. SSL reported leadership roles in Radiosurgery Society and American College of Radiology. The authors have no other conflicts of interest to declare.

Ethical Statement: The authors are accountable for all aspects of the work in ensuring that questions related to the accuracy or integrity of any part of the work are appropriately investigated and resolved.

Open Access Statement: This is an Open Access article distributed in accordance with the Creative Commons AttributionNonCommercial-NoDerivs 4.0 International License (CC BY-NC-ND 4.0), which permits the non-commercial replication and distribution of the article with the strict proviso that no changes or edits are made and the original work is properly cited (including links to both the formal publication through the relevant DOI and the license). See: https://creativecommons.org/licenses/by-nc$\mathrm{nd} / 4.0 \%$.

\section{References}

1 Milano MT, Biswas T, Simone CB 2nd, et al. Oligometastases: history of a hypothesis. Ann Palliat Med 2020. [Epub ahead of print]. doi: 10.21037/apm.2020.03.31.

2 Mihai AM, Rock L, Milano MT. Technical challenges of linac-based stereotactic ablative body radiotherapy: short review for 
non-radiation oncologists. Ann Palliat Med 2020. [Epub ahead of print]. doi: 10.21037/apm-20-950.

3 Dohopolski M, Iyengar P. Oligometastatic non-small cell lung cancer: a narrative review of stereotactic ablative radiotherapy. Ann Palliat Med 2021. [Epub ahead of print]. doi: 10.21037/apm-20-1409.

4 Kent CL, McDuff SGR, Salama JK. Oligometastatic breast cancer: where are we now and where are we headed? - a narrative review. Ann Palliat Med 2020. [Epub ahead of print]. doi: 10.21037/apm-20-1128.

5 Sritharan K, Rieu R, Tree A. A narrative review of oligometastatic prostate cancer-an evolving paradigm. Ann Palliat Med 2021. [Epub ahead of print]. doi: 10.21037/apm-20-1215.

6 Chandy ETJ, Saxby HJ, Pang JW, et al. The multidisciplinary management of oligometastases from colorectal cancer: a narrative review. Ann Palliat Med 2020. [Epub ahead of print]. doi: 10.21037/apm-20-919.

7 Smile TD, Parsai S, Pflederer TM, et al. Treatment paradigms for oligometastatic pediatric cancers: a narrative review with a focus on radiotherapy approaches. Ann Palliat Med 2020. [Epub ahead of print]. doi: 10.21037/apm-20-1023.

8 Chaung KV, Sloan AE, Choi S. Limited brain metastases: a narrative review. Ann Palliat Med 2021. [Epub ahead of print]. doi: 10.21037/apm-21-363.

9 Turchan WT, Chmura SJ. The role of immunotherapy in combination with oligometastasis-directed therapy: a narrative review. Ann Palliat Med 2021. [Epub ahead of print]. doi: 10.21037/apm-20-1528.

$10 \mathrm{Li}$ GJ, Arifin AJ, Al-Shafa F, et al. A review of ongoing trials of stereotactic ablative radiotherapy for oligometastatic disease in the context of new consensus definitions. Ann Palliat Med 2020. [Epub ahead of print]. doi: 10.21037/apm-20-847.

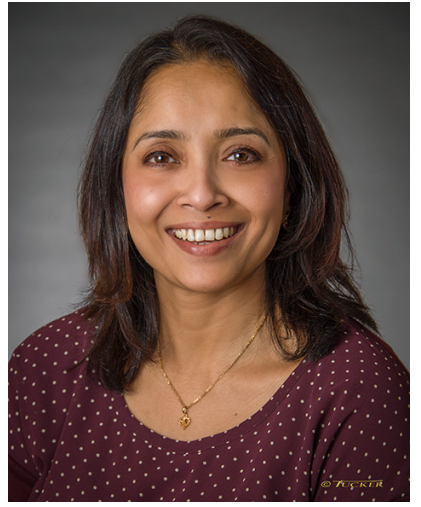

Tithi Biswas

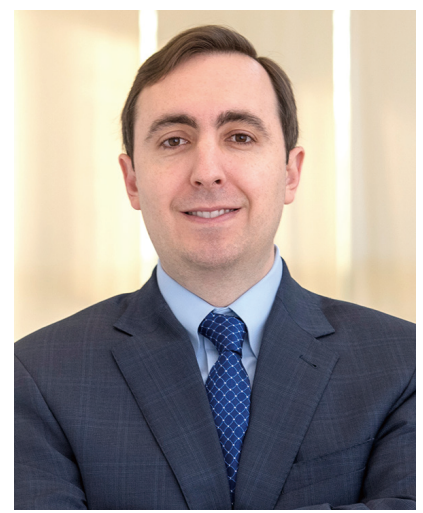

Charles B. Simone II

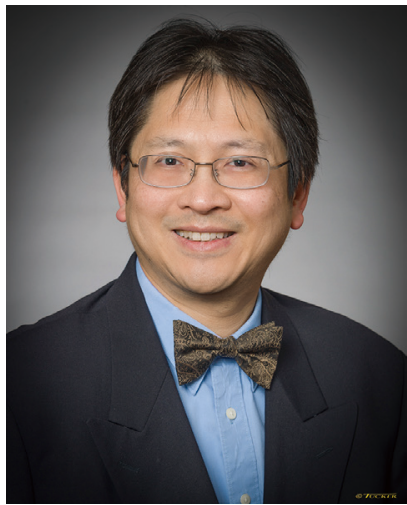

Simon S. Lo

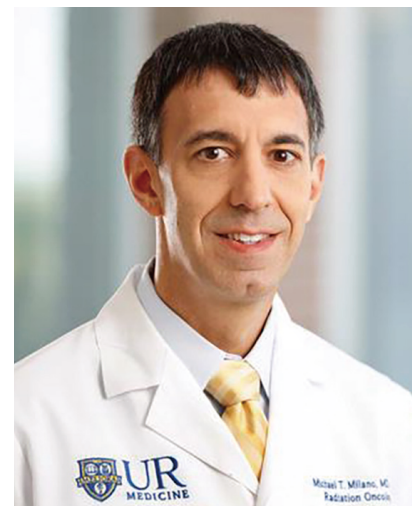

Michael T. Milano 
Tithi Biswas ${ }^{1}$

(Email:Tithi.Biswas@uhbospitals.org)

Charles B. Simone II $^{2,3}$

(Email: csimone@nyproton.com)

Simon S. $\mathrm{Lo}^{4}$, MB, ChB, FACR, FASTRO

(Email: simonslo@uw.edu)

Michael T. Milano

(Email:Michael_Milano@urmc.rochester.edu)

${ }^{1}$ Department of Radiation Oncology, University Hospitals Cleveland Medical Center, Case Western Reserve University, Cleveland, OH, USA;

${ }^{2}$ New York Proton Center, New York, NY, USA;

${ }^{3}$ Memorial Sloan Kettering Cancer Center, New York, NY, USA;

${ }^{4}$ Department of Radiation Oncology, University of Washington School of Medicine, Seattle, WA, USA;

${ }^{5}$ Department of Radiation Oncology, University of Rochester, Rochester, NY, USA.

Submitted Apr 17, 2021. Accepted for publication Apr 29, 2021.

doi: 10.21037/apm-2021-02

View this article at: http://dx.doi.org/10.21037/apm-2021-02

Cite this article as: Biswas T, Simone CB 2nd, Lo SS, Milano MT. Defining the role of curative local therapy in Oligometastatic cancer: a new era. Ann Palliat Med 2021;10(5):5919-5922. doi: 10.21037/apm-2021-02 